J. Phys. IV France 127 (2005) 187-192

(C) EDP Sciences, Les Ulis

DOI: 10.1051/jp4:2005127029

\title{
Influence d'un traitement laser sur les propriétés d'adhésion de surfaces métalliques
}

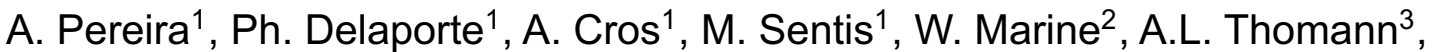 \\ C. Leborgne ${ }^{3}$ et N. Semmar ${ }^{3}$ \\ ${ }^{1}$ LP3, UMR 6182 CNRS, Université de la Méditerranée, Case 917, \\ 13288 Marseille Cedex 9, France \\ ${ }^{2}$ CRMCN, UPR 7251 CNRS, Case 913, 13288 Marseille Cedex 9, France \\ ${ }^{3}$ GREMI, UMR 6606 CNRS, Université d'Orléans, BP. 6744, 45067 Orléans Cedex 2, France
}

\begin{abstract}
Résumé. Nous avons mis en évidence une nanostructuration de la surface due à la formation de nanoparticules d'oxydes métalliques dans le plasma et une modification de propriétés chimiques de la surface permettant l'établissement de liaisons acide-base. Les tests mécaniques d'adhérence ont révélé une qualité d'adhésion tout à fait satisfaisante. Le procédé laser apparaît donc comme une technique «propre » de préparation de surface.
\end{abstract}

\section{INTRODUCTION}

Les surfaces métalliques habituellement utilisées dans l'industrie peuvent être décrites comme des surfaces complexes perturbées sur plusieurs micromètres. On y trouve différents résidus carbonés et oxydes complexes dont l'épaisseur peut aller de quelques centaines d'angströms à quelques micromètres, auxquels il faut ajouter quelques éléments de ségrégation. Leur utilisation nécessite donc le plus souvent un traitement de surface qui a alors pour but : i) d'éliminer les corps étrangers adsorbés (graisses, eau, $\mathrm{CO}_{2}$, poussières), ii) de préparer le substrat pour augmenter la surface de contact et iii) de modifier la chimie de surface pour obtenir une meilleure compatibilité entre les matériaux. Ainsi, si l'on s'intéresse au collage, les techniques actuellement utilisées font appel à des opérations mécano-chimiques à savoir, dégraissage, décapage, phosphatation, suivies de bains d'enduction. Devant des exigences de qualité et de respect de l'environnement de plus en plus sévères, l'industrie a été amenée ces dernières années à envisager des traitements de surface réduisant ou évitant la voie chimique. La mise au point de nouvelles techniques par voie sèche, dites « propres », permettrait ainsi de limiter considérablement les déchets et ouvrirait de nouvelles perspectives de traitement. Dans cette optique, des traitements cryogénique [1] ou par plasma [2,3] ont montré de réelles aptitudes pour la phase de nettoyage mais des problèmes subsistent lorsque l'on s'intéresse à l'adhésion [4,5]. Des travaux réalisés sur des surfaces d'acier ont montré que le procédé laser est une méthode particulièrement attractive [6-8] sans toutefois clairement identifier les causes responsables de la modification des propriétés d'adhérence et expliquer les processus intervenant aux interfaces.

Dans le but de substituer ultérieurement un traitement laser aux opérations mécano-chimiques actuellement utilisées pour préparer les surfaces métalliques, nous nous sommes intéressés au traitement de surfaces d'acier par laser, sans nettoyage préalable tel que l'on peut les trouver dans l'industrie. Le but de notre étude est de démontrer tout d'abord que ce procédé laser peut à la fois nettoyer et modifier les propriétés morphologiques et physico-chimiques de la surface, mais également de corréler ces modifications avec l'amélioration des propriétés d'adhérence et comprendre ainsi les phénomènes responsables de l'amélioration des propriétés d'adhésion. Pour cela, diverses méthodes d'analyse de surface nous ont permis de caractériser les surfaces avant et après traitement laser et parallèlement des essais mécaniques, de type pelage, nous ont permis de caractériser l'efficacité de nos traitements du point de vue de l'adhésion. 


\section{EXPÉRIENCE}

Le matériau utilisé lors de cette étude est un acier faiblement allié à $0.12 \%$ de carbone (1C selon NF A 36-301). La source laser utilisée est un laser à excimères XeCl (EMG 203 MSC) de durée d'impulsion de $25 \mathrm{~ns}$ et émettant dans l'UV à $308 \mathrm{~nm}$. Les traitements ont été réalisés à température ambiante et pression atmosphérique avec des fluences variant entre 5 et $10 \mathrm{~J} / \mathrm{cm}^{2}$. Les échantillons étant recouverts d'une pellicule d'huile, une buse d'aspiration placée dans l'axe du faisceau nous a permis de récupérer les composés hydrocarbonés. Les échantillons étaient fixés sur un système de tables motorisées, pilotées par ordinateur, afin de traiter uniformément la totalité de la surface.

Les surfaces ont été caractérisées du point de vue chimique (XPS) et morphologique (MEB). L'XPS nous a fourni une analyse qualitative et quantitative de la couche superficielle, typiquement 9-10 nm (soit 3 fois le libre parcours moyen des photoélectrons) alors que les analyses MEB nous ont permis d'obtenir une image de la topographie de surface et de détecter la présence de pores, fissures ou autres.

Parallèlement, des expériences d'imagerie rapide, utilisant une camera CCD intensifiée, ont été réalisées afin d'étudier la formation et l'évolution temporelle du plasma d'ablation au-dessus de la surface. Enfin, une étude de la dynamique de dépôt de nanoparticules sur la surface a été réalisée à l'aide du dispositif expérimental présenté figure 1. Le faisceau $\mathrm{XeCl}$ permet de traiter la surface alors qu'un faisceau d'argon continu $(491 \mathrm{~nm})$ vient sonder le redépôt ce qui revient à faire de la spectroscopie d'absorption. Pour cela, nous avons placé une lame de verre près de l'impact laser et parallèle à la surface d'acier. Le faisceau du laser Argon est focalisé sur la lame de verre à 1 mm du bord de l'impact. Il passe ensuite dans un spectromètre pour sélectionner la longueur d'onde à $491 \mathrm{~nm}$, puis il est collecté en sortie par une photodiode intensifiée. Après s'être affranchi des perturbations imputables au plasma, ce dispositif nous permet de suivre la dynamique de redépôt après chaque tir.

Une fois la surface traitée, la phase d'enduction effectuée et le caoutchouc vulcanisé sur la surface, les assemblages ainsi réalisés ont été testés mécaniquement à l'aide d'un test de pelage (norme ASTM D429). Ces tests ont été réalisés dans trois conditions environnementales différentes : i) à l'état neuf, c'est à dire après la phase de vulcanisation, ii) après vieillissement par immersion dans l'eau bouillante $(40 \mathrm{H})$, et iii) après vieillissement dans une atmosphère de brouillard salin $(1000 \mathrm{H})$. L'analyse des faciès

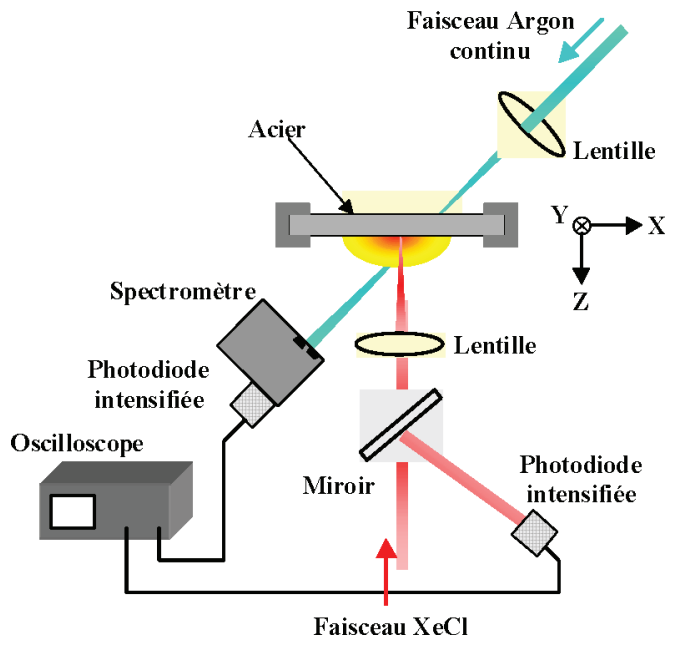

Figure 1. Dispositif expérimental pour l'étude de la dynamique de redépôt. 
de rupture nous a permis d'identifier les zones de rupture. Un essai est considéré comme satisfaisant au regard de la préparation de surface si le faciès de pelage n'est pas de type interfaciale métal - couche d'enduction.

\section{RÉSULTATS EXPÉRIMENTAUX}

Les analyses XPS des échantillons bruts ont révélé la présence de fer, d'oxygène, de carbone ainsi que d'autres éléments tels que du zinc, du calcium et parfois du manganèse. Le pourcentage important de carbone (46\% malgré un dégraissage à l'acétone pour éviter de polluer la chambre d'analyse), attribué aux résidus d'huile et à la pollution atmosphérique, ainsi que la présence de zinc et de calcium, indique une contamination importante. Après traitement laser, l'élimination complète de $\mathrm{Zn}$, Ca et la diminution significative de la quantité de carbone (29\%) en surface confirment l'effet de nettoyage par traitement laser.

Les spectres XPS $\mathrm{O}_{1 \mathrm{~s}}$ sont présentés sur la figure 2. Avant traitement (Fig.2a), le spectre $\mathrm{O}_{1 \mathrm{~s}}$ est composé de quatre pics attribués aux contributions de l'oxygène provenant respectivement des liaisons $\mathrm{Fe}-\mathrm{O}$, des groupes hydroxyle $-\mathrm{OH}$, de l'eau absorbée et pour finir de composés carbonés absorbés. La déconvolution des spectres $\mathrm{O}_{1 \mathrm{~s}}$ obtenus après traitement laser (Fig.2b) montre la disparition des composantes attribuées à $\mathrm{H}_{2} \mathrm{O}$ et aux composés carbonés. Les spectres $\mathrm{O}_{1 \mathrm{~s}}$ sont alors constitués par deux composantes attribuées aux états de liaison de l'oxygène $\mathrm{Fe}-\mathrm{O}$ et $-\mathrm{OH}$. Les analyses quantitatives montrent d'autre part une diminution de la contribution des groupements -OH d'autant plus importante que la fluence laser augmente. Ces résultats indique très clairement une oxydation de la surface, l'utilisation d'une fluence élevée favorisant la formation de $\mathrm{Fe}_{2} \mathrm{O}_{3}$ au détriment de $\mathrm{FeOOH}$.

Nous avons donc mis en évidence qu' au travers des traitements laser, il est à la fois possible de nettoyer la surface mais aussi de former de nouveaux oxydes que l'on observe sous forme de nanoagrégats et qui viennent structurer la surface (Fig.3). La formation de ces nanoagrégats est directement liée à la formation de nanoparticules d'oxyde dans le plasma d'ablation et qui se deposent sur la surface par rétrodiffusion.

Des expériences d'imagerie rapide nous ont permis d'étudier la formation et l'expansion du plasma au cours du temps (Fig. 4). Le plasma reste dans les premiers instants confiné à la surface, puis il s'expand axialement, latéralement et enfin on observe l'apparition de deux lobes de part et d'autre de

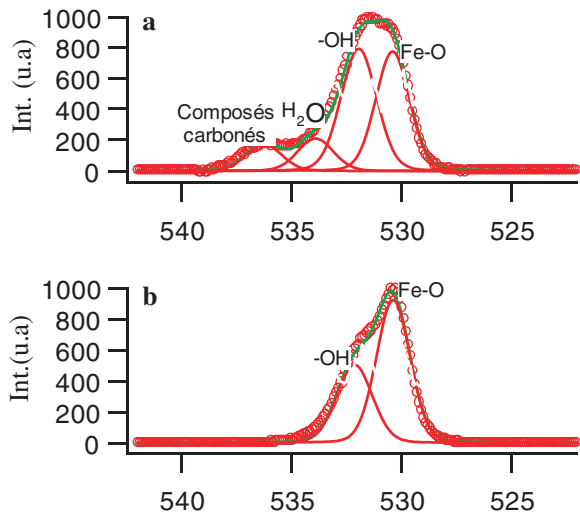

Energie de liaison $(\mathrm{eV})$

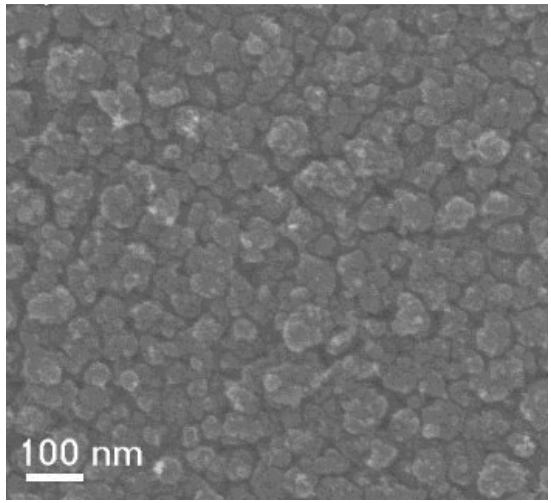

Figure 3. Image MEB de la morphologie de surface: nanostructuration de la surface par traitement laser.
Figure 2. Spectres XPS $\mathrm{O}_{1 \mathrm{~s}}$ déconvolués avant (a) et après (b) traitement laser. 


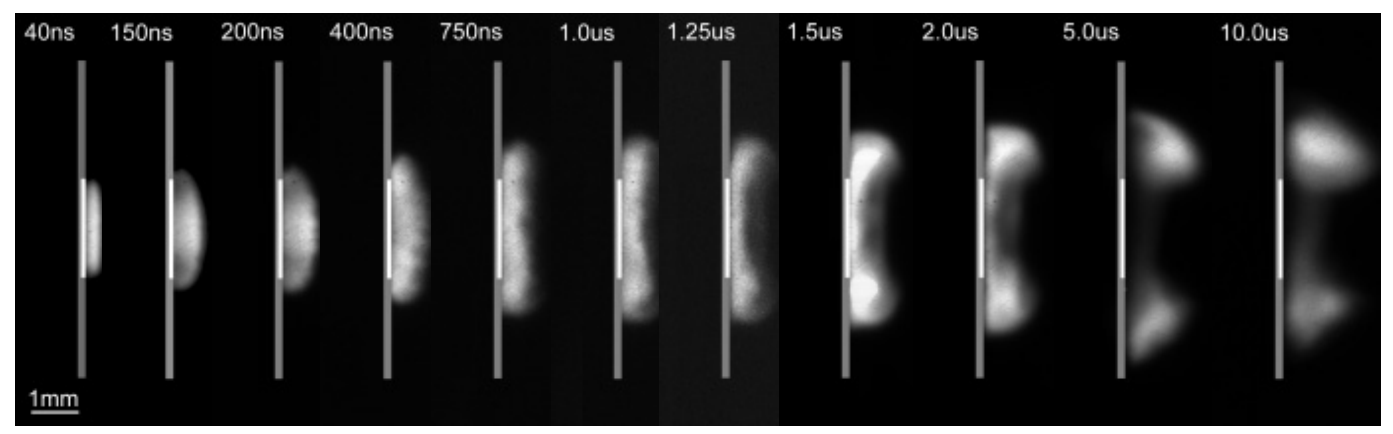

Figure 4. Evolution temporelle du plasma d'ablation de l'acier à pression atmosphérique.

l'impact laser. Cette forme particulière d'expansion du plasma est directement liée à la pression du gaz environnant. Dans les premiers instants, nous sommes en présence d'un plasma dense confiné à la surface, ce qui a pour effet de déplacer les molécules de gaz. Ces molécules déplacées sont alors compressées sur le front d'expansion, ce qui freine et stoppe l'expansion axiale. Celle-ci se fait alors latéralement ( $\mathrm{t} \geq 250 \mathrm{~ns}$ ) car la différence de pression y est plus faible. On retrouve alors les mêmes phénomènes jusqu'à ce que l'expansion latérale soit stoppée. A cet instant, la différence de pression entre le front d'expansion et le centre du plasma (plus d'apport par vaporisation) est relativement élevée. Il y a alors formation d'un phénomène de vortex en périphérie qui se matérialise par l'apparition de deux lobes. L'accumulation d'espèces sur la périphérie du plasma a également été mise en évidence expérimentalement et numériquement [9] pour des pressions de gaz ambiant plus faibles. La formation d'un vortex, drainant les particules de vapeur métallique sur les bords du plasma serait une explication possible.

Ces mesures d'imagerie donnent une vue globale de l'expansion temporelle du plasma, mais il difficile d'en tirer des conclusions définitives sur la formation de nanoparticules. On peut cependant supposer que les parties les plus intenses correspondent à des zones où la densité d'espèces est la plus élevée et donc où la probabilité de collisions, et de formation de nanoparticules, est la plus importante.

Nous avons de plus pu corréler l'expansion latérale maximale du plasma avec la distance de redépôt de nanoparticules autour de l'impact laser et ainsi observer des distances de l'ordre de $2 \mathrm{~mm}$ dans les deux cas. Ce processus de redépôt de nanoparticules a été étudié plus en détail à l'aide du dispositif expérimental présenté figure 1. Il s'agit en faite de spectroscopie d'absorption du redépôt puisque celui-ci fait office de filtre en absorbant une partie de l'énergie incidente du faisceau du laser Argon.

La figure 5 présente l'évolution temporelle de l'intensité relative du laser Argon mesurée après la lame de verre sur laquelle s'effectue le redépôt. Nous avons ainsi pu estimer le début du processus de dépôt sur la lame de verre, c'est-à-dire pour des temps compris entre 950 et 1015 ns (Fig.5a). La transmission décroissante du signal pendant plus de $50 \mu$ s correspond ensuite à une épaisseur de film croissante (Fig.5b). Ces résultats sont en accord avec les expériences d'imagerie rapide, puisque nous constatons une disparition significative de l'intensité lumineuse du plasma pour un délai de $50 \mu \mathrm{s}$.

Afin de vérifier l'efficacité de nos traitements vis-à-vis des propriétés d'adhérence, des tests de pelage ont été réalisés avec des éprouvettes traitées par laser. Nous avons ensuite comparé ces résultats avec ceux obtenus avec des éprouvettes traitées mécano-chimiquement (Tab. 1).

Quelles que soient les conditions de vieillissement, un traitement laser permet de garantir des faciès de rupture cohésifs dans la masse du caoutchouc (100R), ce qui témoigne de liaisons interfaciales suffisamment fortes ne permettant pas la diffusion de l'eau à l'interface métal-caoutchouc 

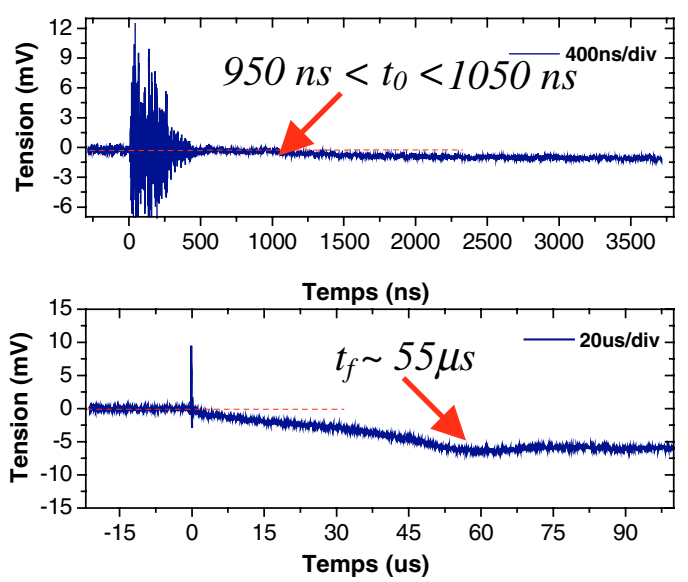

Figure 5. Dynamique de redépôt des nanoparticules sur la surface.

lors du vieillissement. Si l'on compare avec les traitements mécano-chimiques, on constate de meilleurs résultats aux tests, puisque que ce soit pour un cycle DGD ou DGD+phosphatation des ruptures à l'interface métallique sont observées. On peut donc dire que le traitement laser regroupe les trois fonctions des traitements mécano-chimiques : i) l'aspect nettoyage avec l'élimination des composés carbonés, ii) l'aspect grenaillage qui modifie la rugosité et la chimie de surface, iii) l'aspect phosphatation, la protection contre la corrosion étant meilleure avec les traitements laser.

Tableau 1. Résultats des tests de pelage après traitement mécano-chimique (DGD : cycle dégraissage-grenaillagedégraissage, avec ou sans phosphatation) et laser. 100R signifie une rupture 100\% cohésive dans le caoutchouc, 70R30M une rupture de type interfaciale dont $30 \%$ se situe à l'interface métallique.

\begin{tabular}{lccc}
\hline \hline & \multicolumn{2}{c}{ Mecano-chimique } & \\
\cline { 2 - 3 } & DGD & DGD+Phosphatation & Traitement laser \\
\hline Etat Neuf & $100 \mathrm{R}$ & $100 \mathrm{R}$ & $100 \mathrm{R}$ \\
Eau bouillante $(40 \mathrm{H})$ & $100 \mathrm{R}$ & $100 \mathrm{R}$ & $100 \mathrm{R}$ \\
Brouillard salin $(1000 \mathrm{H})$ & $45 \mathrm{R} 55 \mathrm{M}$ & $70 \mathrm{R} 30 \mathrm{M}$ & $100 \mathrm{R}$ \\
\hline \hline
\end{tabular}

\section{DISCUSSION ET CONCLUSION}

Cette étude nous a permis de corréler les modifications des propriétés de surface induites lors des traitements laser, avec les propriétés d'adhérence et d'identifier trois paramètres importants dans les processus d'adhésion. Tout d'abord le nettoyage de la surface avec l'élimination des divers polluants, moins il y a de contamination, meilleure sera la tenue des assemblages. Ensuite, l'oxydation de la surface qui favorise la création de nouvelles liaisons chimiques. En effet, l'oxydation se traduit par une augmentation du caractère acido-basique de la surface. On a alors formation de liaisons de type acide de Lewis avec les cations métalliques et de liaisons de type base de Brönsted avec les groupes hydroxyle $-\mathrm{OH}$. Enfin le troisième paramètre est la nanostructuration de surface qui de part sa porosité favorise l'ancrage mécanique et donc l'adhésion.

Nous avons d'autre part mis en évidence que ce phénomène de nanostructuration est directement lié à la formation d'un plasma d'ablation et à la formation de nanoparticules dans le plasma qui se déposent sur la surface par rétrodiffusion. L'étude de la dynamique de dépôt a en particulier révélé un processus ayant une durée supérieure à $50 \mu \mathrm{s}$. 


\section{Bibliographie}

[1] D.M. Brewis, G.W. Critchlow, C.A. Curtis, Int. J. Adhes. Adhes. 19 (1999) 253

[2] W. Leahy, V. Barron, M. Buggy, T. Young, A. Mas, F. Schue, T. Mc Cabe, M. Bridge, J. Adhes. 77 (2001) 215

[3] K.Y. Rhee, N.S. Choi, S.J. Park, J. Adhes. Sci. Technol. 16 (2002) 1487

[4] E. Darque-Ceretti, E. Felder, Adhésion et adhérence (Edition CNRS, Paris, 2003), p. 339

[5] V. Monna, Réactivité des décharges en flux dans des mélanges de gaz pour le traitement des surfaces, Thèse de doctorat, Université Toulouse III - Paul Sabatier, 2002

[6] H.R. Jahani, B. Moffat, R.E. Mueller, D. Fumo, W. Duley, T. North, B. Gu, Appl. Surf. Sci. 127-129 (1998) 767

[7] R. Broad, J. French, J. Sauer, Int. J. Adhes. Adhes. 19 (1999) 193

[8] G.W. Critchlow, C.A. Cottam, D.M. Brewis, D.C. Emmony, Int. J. Adhes. Adhes. 17 (1997) 143

[9] T.E. Itina, J. Hermann, Ph. Delaporte, M. Sentis, Phys. Rev. E 66 (2002) 066406 